\title{
Effects of salt stress on low molecular antioxidants and redox state of plastoquinone and P700 in Arabidopsis thaliana (glycophyte) and Eutrema salsugineum (halophyte)
}

\author{
M. WICIARZ ${ }^{*}$, E. NIEWIADOMSKA ${ }^{* *+}$, and J. KRUK* \\ Department of Plant Physiology and Biochemistry, Faculty of Biochemistry, Biophysics and Biotechnology, \\ Jagiellonian University, Gronostajowa 7, 30-387 Kraków, Poland ${ }^{*}$ \\ The Franciszek Górski Institute of Plant Physiology, Polish Academy of Sciences, Niezapominajek 21, \\ 30-239 Kraków, Poland**
}

\begin{abstract}
The effects of $\mathrm{NaCl}$ treatment were analysed in two species of considerably different resistance. In glycophyte, the content of ascorbate decreased but lipophilic antioxidants ( $\alpha$-tocopherol, plastochromanol, and hydroxy-plastochromanol) increased due to $150 \mathrm{mM} \mathrm{NaCl}$. In halophyte, $300 \mathrm{mM} \mathrm{NaCl}$ caused a significant increase in hydrophilic antioxidants (ascorbate, total glutathione) but not in the lipophilic antioxidants. The redox states of plastoquinone (PQ) and P700 were also differently modulated by salinity in both species, as illustrated by an increased oxidation of these components in glycophyte. The presented data suggest that E. salsugineum was able to avoid a harmful singlet oxygen production at PSII, which might be, at least in part, attributed to the induction of the ascorbate-glutathione cycle. Another important cue of a high salinity resistance of this species might be the ability to sustain a highly reduced states of PQ pool and P700 under stress, which however, drastically affect the NADPH yield.
\end{abstract}

Additional key words: chloroplast; oxidative stress; photosystems; salinity.

\section{Introduction}

Salinity (in the general sense) causes disturbance in natural balance in plant cells, as well as, in the redox and energy status which affect cellular metabolism and result in less efficient photosynthesis (Takahashi and Murata 2008, Suzuki et al. 2012). Chloroplasts are particularly subjected to salt stress because of their sensitivity to external changes (Pfalz et al. 2012). Uptake of excessive sodium ions into cells disturbs the osmotic balance and water availability, which results in stomatal closure and decrease in the $\mathrm{CO}_{2} / \mathrm{O}_{2}$ ratio (Munns and Tester 2008). As a consequence, overreduction of photosynthetic electron transport (PET) occurs which leads to unbalanced ATP/NADPH ratio (Lokhande et al. 2011). Excess of electrons is transferred to molecular oxygen that is reduced to highly active species, namely, superoxide anion radical, $\mathrm{H}_{2} \mathrm{O}_{2}$, and

hydroxyl radical, which are produced mostly on the acceptor site of PSI (Mehler reaction), at PSII (at the $\mathrm{Q}_{\mathrm{B}}$ site or via cytochrome $\left.b_{559}\right)$ or at the plastoquinone pool (PQ-pool) (Pospíšil 2009, Khorobrykh et al. 2015). Furthermore, energy transfer from triplet state of $\mathrm{P}_{680}$ to molecular oxygen produces singlet oxygen in PSII. Although the leakage of electrons contributes to reactive oxygen species (ROS) production, it allows to control the redox poise of electron carriers (Noctor and Foyer 1998). In addition, a blockage of $\mathrm{CO}_{2}$ flow to stomata also influences the Calvin cycle through depletion of 3-phosphoglyceric acid and $\mathrm{NADP}^{+}$availability. The shortage of the latter contributes to ROS production, especially of $\mathrm{H}_{2} \mathrm{O}_{2}$, which is known to inhibit synthesis of D1 protein (Takahashi and Murata 2006, Murata et al. 2007).

Received 28 December 2016, accepted 10 April 2017, published as online-first 8 May 2017.

${ }^{+}$Corresponding author; e-mail: e.niewiadomska@ifr-pan.edu.pl

Abbreviations: NPQ - nonphotochemical quenching; PC-8 - plastochromanol-8; PET - photosynthetic electron transport; PQ plastoquinone; ROS - reactive oxygen species; Toc - tocopherol; $\mathrm{Y}_{\mathrm{I}}$ - the quantum efficiency of PSI, $\mathrm{Y}_{\mathrm{ND}}$ - donor-side limitation to PSI; Y $\mathrm{NA}$ - acceptor-side limitation to PSI.

Acknowledgements: The Jagiellonian University is a partner of the Leading National Research Center (KNOW) supported by the Ministry of Science and Higher Education. This work was supported by Polish National Science Centre project (2011/03/B/NZ9/01619, EN and MW).

Contributions: M. Wiciarz and E. Niewiadomska performed most of the experiments and wrote the manuscript. J. Kruk and M. Wiciarz performed HPLC experiments and contributed to the preparation of text and figures.

(C) The Author(s). This article is published with open access at link.springer.com 
In order to minimize these negative effects and restore a disturbed homeostasis, plants activate short and longterm mechanisms in order to adjust the photosynthetic apparatus to new demands and activate acclimation responses (Scheibe et al. 2005, Dietzel et al. 2008). All these mechanisms, such as energy dissipation pathways of the PET chain (i.e. nonphotochemical quenching, state transitions, cyclic and pseudocyclic electron transport) or pathways involving cell compartments (i.e. malate valve, photorespiration or alternative oxidase pathway), allow to optimize electron flow and a required rate of the production of reducing equivalents for metabolic processes during stress conditions (Scheibe et al. 2005). Redox imbalance and subsequently ROS production is also sensed by the antioxidant systems. The enzymatic responses are mostly generated through the water-water cycle enzymes (superoxide dismutase, SOD, ascorbate peroxidase, APX, monodehydroascorbate reductase, MDHAR, dehydroascorbate reductase, DHAR, and glutathione reductase, GR) and redox regulatory enzymes and compounds, such as NADPH-dependent thioredoxin reductase (NTRC), 2-Cys peroxiredoxin (2-Cys Prx), thioredoxins or ferredoxin-thioredoxin reductase (Foyer and Noctor 2009). The second important target group are low molecular antioxidants, such as ascorbate, glutathione, prenyllipids, flavonoids, and carotenoids (Apel and Hirt 2004). Ascorbate and glutathione are redox buffers that have multiple functions in plants and serve as powerful antioxidants reacting directly with singlet oxygen, superoxide, and hydroxyl radical or as a reducing substrate for $\mathrm{H}_{2} \mathrm{O}_{2}$ detoxification (Foyer and Noctor 2011). In the latter case, it prevents overreduction of PET by regulating electron flow and production of photosynthetic assimilatory power and decrease the risk of singlet oxygen formation (Noctor and Foyer 1998). Ascorbate plays also an important role as a secondary antioxidant by reducing the oxidized form of $\alpha$-tocopherol, thereby limiting lipid peroxidation in chloroplasts (Munné-Bosch 2005). Among prenyllipids anitoxidants, the best known are tocopherols, plastochromanol, and plastoquinol (Munné-Bosch and Alegre 2002, Nowicka and Kruk 2010, Kruk et al. 2016). These lipophilic compounds are able to prevent lipid peroxidation or deactivate singlet oxygen (Munné-Bosch 2005, Gruszka et al. 2008, Nowicka and Kruk 2010).

Although ROS interact with many important cellular components, they function as signalling molecules (Noctor and Foyer 1998). Moreover, glutathione and ascorbate are not only essential to annihilate ROS but can also act as signal transductors initiating signalling cascades towards changes in nuclear gene expression (Baier and Dietz 2005, Noctor 2006, Scheibe et al. 2005). The main redox sensor in chloroplast is the PQ-pool (Pfannschmidt et al. 2009). This pool is composed of the fractions photosynthetically active (localized mainly in thylakoid membranes) and inactive (present in plastoglubules). The inactive part, owing to its antioxidant properties, contributes to scavenging of singlet oxygen and superoxide anion radical (Szymańska and Kruk 2010). It has been shown that the amount and size of plastoglobules increase during salt stress (Hernandez et al. 1995). The redox state of the PQpool regulates many processes, such as state transitions, expression of PSII and PSI proteins, SOD, APX, and others (Pfannschmidt 2003, Ślesak et al. 2003, Chang et al. 2004, Puthiyaveetil et al. 2012).

One of many approaches to study salt defense mechanisms is the use of halophytic plants. Several studies pointed to a constitutive preparedness of these plants to salt conditions (Taji et al. 2004, Amtmann 2009, Dassanayake et al. 2011). In these plants, several protecting strategies are activated in order to stabilize photosystems, maintain the rate of photosynthesis, secure Calvin cycle enzymes, and modulate redox state of many components (Niewiadomska and Wiciarz 2015). Moreover, halophytes seem to avoid excessive ROS production by a more pronounced ability to activate and regulate antioxidant response. By contrast to glycophytes, this response is more intense and rapid, allowing to control ROS production at a certain threshold (Ellouzi et al. 2011, Ozgur et al. 2013, Bose et al. 2014). Likewise, in glycophytic Arabidopsis thaliana, a strong mobilization is observed at the level of stress hormones, transcription of stress genes, as well as photosynthetic performance, due to salinity treatment with 100-200 mM NaCl. Whilst, in glycophytic Eutrema salsugineum, a very weak change in these stress markers was found under a much higher salinity levels (Gong et al. 2005, Stepien and Johnson 2009, Wiciarz et al. 2015, Pilarska et al. 2016). Lokhande et al. (2011) suggested that changes in the redox and energetic status in halophytes accounts for their tolerance to salinity. However, in this type of analysis much attention is directed toward enzymatic response to salt treatments. Less emphasis is placed on low molecular antioxidants, although their role in a stress defence is crucial (Bose et al. 2014).

In our previous study, we have shown enhanced PSII electron transport rate in Eutrema salsugineum (Thellungiella salsuginea) which was accompanied by pronounced $\mathrm{H}_{2} \mathrm{O}_{2}$ production from the PQ-pool and increased chlororespiration (Wiciarz et al. 2015). A goal of this study was to verify hypothesis that a considerable difference between the halophyte and glycophyte in response to salinity stress, as previously reported, is related to the engagement of soluble nonenzymatic antioxidants. Considering the above-mentioned phenomenon of high $\mathrm{H}_{2} \mathrm{O}_{2}$ generation in chloroplasts of halophyte, we also aimed to characterize a lipophilic antioxidants, which are localised close to PSII and may modify the functioning of the overall photosynthetic electron transport. 


\section{Materials and methods}

Plant material and growth conditions: Arabidopsis thaliana (Col-0) and Eutrema salsugineum (Thellungiella salsuginea) ecotype Shandong were used for all experiments. Seeds were obtained from the Nottingham Arabidopsis Stock Centre, UK. Growth conditions were as those described earlier (Wiciarz et al. 2015). After development of leaf rosette, four-week-old $A$. thaliana and E. salsugineum plants were daily irrigated with $\mathrm{NaCl}$ solutions $(0.15 \mathrm{M}$ for $A$. thaliana and $0.3 \mathrm{M}$ for E. salsugineum), while watered plants served as controls. These two $\mathrm{NaCl}$ concentrations were chosen on basis of earlier reports describing stress responses of this two species under a broad salinity range (Vinocur and Altman 2005, Gong et al. 2005, Stepien and Johnson 2009). They revealed that a much stronger salinity treatment is needed to evoke stress effects in halophyte than in glycophyte. Chosen concentrations also correspond with our earlier characteristic of changes in chloroplast metabolism in these two species (Wiciarz et al. 2014, Pilarska et al. 2016). After $7 \mathrm{~d}$ of $\mathrm{NaCl}$ treatment, the plants were collected and used for further experiments.

Ascorbate and total glutathione in leaves: The ascorbate content was determined according to Takahama and Oniki (1992). Fresh leaves (2-3) were ground in a mortar with $10 \mathrm{ml}$ of $6 \%(\mathrm{w} / \mathrm{v})$ metaphosphoric acid solution with addition of $0.5 \mathrm{mM}$ diethylenetriaminepentaacetic acid (DTPA) and at the proportion of $500 \mu 1$ of metaphosphoric acid solution for $50 \mathrm{mg}$ of leaf fresh mass (FW). The homogenate was centrifuged at $4^{\circ} \mathrm{C}$ for $4 \mathrm{~min}$ at $10,000 \times g$. The supernatant was transferred to an Eppendorf tube and carefully neutralised with $2.5 \mathrm{M}$ potassium carbonate. Ascorbate concentration in the probes was determined by test stripes (Merckoquant, Merck KGaA, Darmstadt, Germany) and reflectometers (RQflex, Merck KGaA, Darmstadt, Germany) according to the producer's instruction, and compared with ascorbate standards.

Total glutathione content was measured according to Luwe et al. (1993). The samples were prepared in the same way as for ascorbate measurements and the supernatants were used in a further procedure. The reaction mixture consisted of $100 \mathrm{mM}$ potassium phosphate buffer, $\mathrm{pH}$ 7.5, containing $6.3 \mathrm{mM}$ ethylenediaminetetraacetic acid

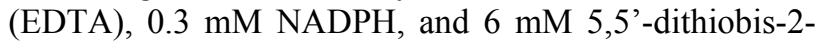
nitrobenzoic acid (DTNB). The reaction was initiated by

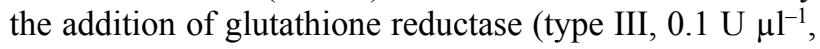
Sigma-Aldrich, Poznan, Poland) into the cuvette (1 ml) and the increase of absorbance at $412 \mathrm{~nm}$ (DU-640, Beckman Instruments Inc., Brea, USA) was measured and compared with GSH standard. Control rates in the absence of the extract were subtracted.

Prenyllipids content and the redox state of total plastoquinone: The concentration of tocochromanols ( $\alpha$-Toc, PC-8), the total (oxidized plus reduced) plastoquinone
(PQ) and its redox state in leaves was determined according to Kruk and Karpinski (2006). In general, three to four leaves were homogenized in a cold mortar with $750 \mu \mathrm{l}$ of cold ethyl acetate, then next $750 \mu \mathrm{l}$ of ethyl acetate was added, and homogenization continued. The extract $(400 \mu \mathrm{l})$ was transferred to an Eppendorf tube and immediately evaporated to dryness under stream of nitrogen. Afterwards, the extract was dissolved in $200 \mu \mathrm{l}$ of methanol/hexane $(340 / 20, \mathrm{v} / \mathrm{v})$ solvent, shortly centrifuged, and $100 \mu \mathrm{l}$ of the extract was analysed by HPLC system, composed of $P U-980$ pump and UV-VIS detector system UV-970 (Jasco Inc., Tokyo, Japan), and fluorescence detector RF10-AXL (Shimadzu, Kyoto, Japan). The concentration of PQ redox forms, as well as tocochromanols, was determined from the area of respective peaks and were compared to standards. The measurements were performed using reversed-phase $\mathrm{C}_{18}$ column (Nucleosil 100, $250 \times 4 \mathrm{~mm}, 5 \mu \mathrm{m}$, Teknokroma, Barcelona, Spain) with the flow rate of $1.5 \mathrm{ml} \mathrm{min}{ }^{-1}$ and absorption detection at $255 \mathrm{~nm}$ for prenylquinones and fluorescence detection at $\lambda_{\mathrm{ex}}=290 \mathrm{~nm}, \lambda_{\mathrm{em}}=330 \mathrm{~nm}$ for chromanols. PC-OH was determined as described previously (Szymańska et al. 2014).

Chlorophyll (Chl): The total $\mathrm{Chl}$ content was determined spectrophotometrically (DU-640, Beckman Instruments Inc., Brea, USA) and calculated according to Lichtenthaler (1987). Ethyl acetate extract $(400 \mu \mathrm{l})$ was evaporated to dryness under stream of nitrogen and dissolved in $2 \mathrm{ml}$ of methanol.

Nonphotochemical quenching and PSI parameters: Nonphotochemical quenching (NPQ) and PSI parameters were determined with Dual PAM (Heinz Walz GmbH, Effeltrich, Germany). Due to technical limitations, measurements were done on cut-off leaves dark-adapted for 15-20 min. To reach a steady state of electron transport an illumination with red actinic irradiation of 126 $\mu \mathrm{mol}\left(\right.$ quantum) $\mathrm{m}^{-2} \mathrm{~s}^{-1}$ for $5 \mathrm{~min}$ was used. NPQ and PSI parameters were calculated according to Bilger and Björkman (1990) and Klughammer and Schreiber (1994), respectively.

NADP-MDH activity and NADPH in leaves: The activity of chloroplast NADP-dependent malate dehydrogenase (NADP-MDH; EC 1.1.1.82) was assayed according to Scheibe and Stitt (1988) with minor modifications. The powdered leaves were homogenized in $0.1 \mathrm{M}$ Tris$\mathrm{HCl}$ buffer, $\mathrm{pH}$ 8.0, containing $1 \mathrm{mM}$ EDTA, $5 \mathrm{mM}$ DTT, and $0.5 \%$ PVP-40. Homogenates were centrifuged for $5 \mathrm{~min}$ at $13,300 \times g$ and the supernatant was used for measurements. Extracts of soluble proteins were incubated for $15 \mathrm{~min}$ in $0.1 \mathrm{M}$ Tris-HCl buffer, $\mathrm{pH} 8$, containing $1 \mathrm{mM}$ EDTA, $20 \mathrm{mM}$ DTT, and $0.2 \mathrm{mM}$ NADPH. Decrease of absorbance at $340 \mathrm{~nm}$ (DU-640, Beckman 
Instruments Inc., Brea, USA) was followed for 7 min after starting the reaction by addition of $1 \mathrm{mM}$ oxaloacetate. Activity of NADP-MDH was expressed in nmol of consumed NADPH per mg protein per min.

The concentration of NADPH was measured by Amplite Colorimetric NADP/NADPH Assay Kit (AAT Bioquest Inc., Sunnyvale, USA) according to manufacturer's instructions. Fifty $\mathrm{mg}$ of leaves were ground in a mortal with $1 \mathrm{ml}$ of PBS buffer, centrifuged in a cold benchtop for $5 \mathrm{~min}$ at $10,000 \times g$ and stored on ice. The

\section{Results}

Effect of moderate salinity on low molecular antioxidants: To have an insight into the antioxidant response to mild salinity treatment, we compared low molecular antioxidants, such as ascorbate, glutathione, $\alpha$-Toc, and PC-8. The analysis of hydrophilic antioxidants (Fig. 1A,B) involved in the ascorbate-glutathione cycle revealed that in E. salsugineum, a significant increase of ascorbate and total glutathione concentration were found after salinity. In supernatant was incubated for $45 \mathrm{~min}$ in the reaction mixture at room temperature, then absorbance was measured at $575 \mathrm{~nm}$ (DU-640, Beckman Instruments Inc., Brea, USA) and compared with NADPH standard.

Statistical analysis: Significances between the means were analysed with the Student's $t$-test. Significant differences are marked at $P \leq 0.05$. Calculations were made with MS Excel 2013.

turn, concentration of ascorbate decreased in A. thaliana, while that of glutathione remained unaffected. In the case of hydrophobic antioxidants, an opposite effect was observed (Fig. $1 C-E$ ). In leaves of E. salsugineum, the concentrations of $\alpha$-Toc and PC- 8 were not affected significantly after salt treatment. In contrast, in $A$. thaliana the content of these compounds increased by 50 and $60 \%$ after $\mathrm{NaCl}$ treatment, respectively. In the control

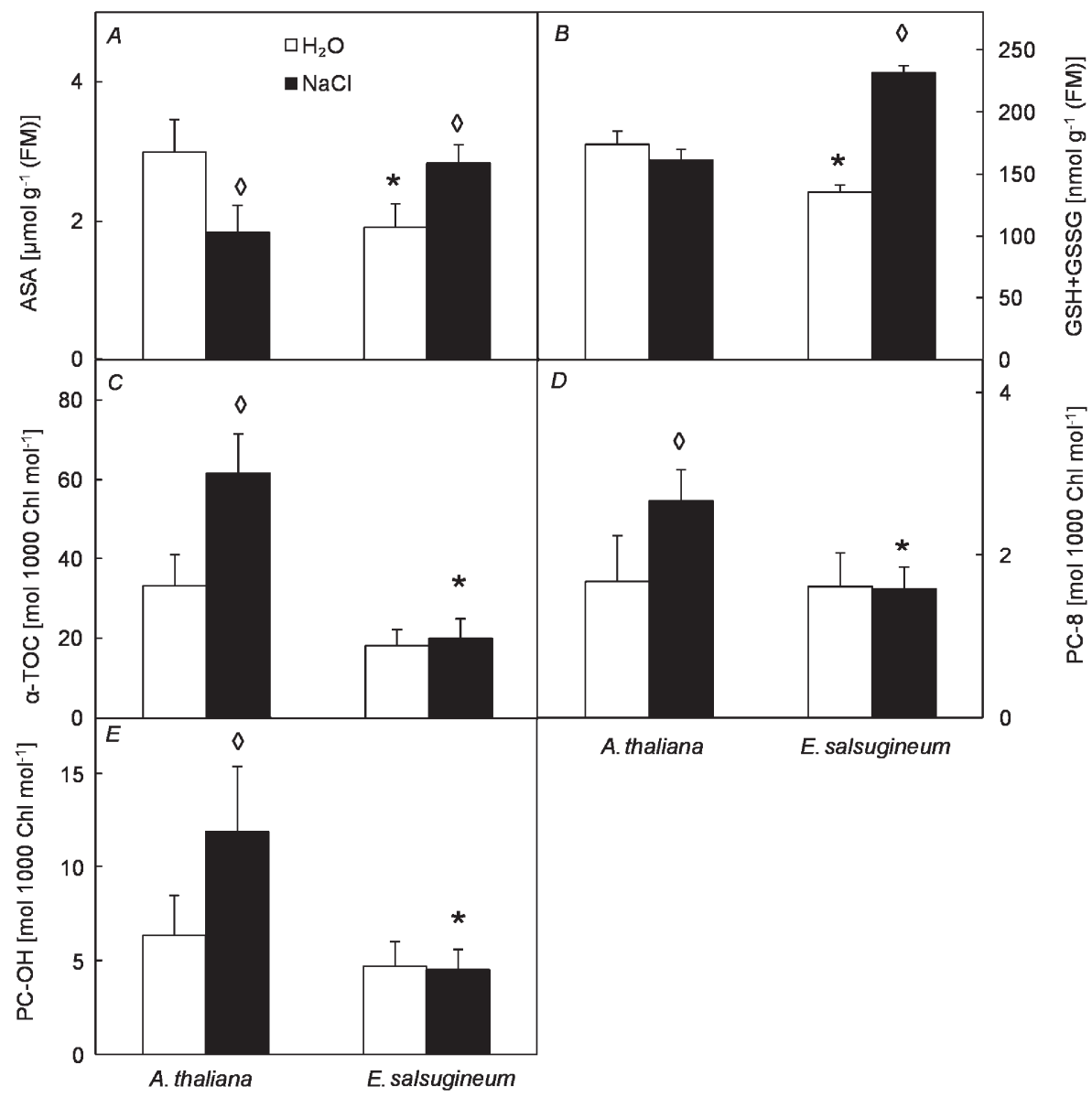

Fig. 1. The content of ascorbate $(A)$, total glutathione content $(B)$, the content of $\alpha$-tocopherol $(\alpha$-Toc; $C)$, plastochromanol (PC-8; $D)$, and hydroxyplastochromanol (PC-OH; E) in leaves of Arabidopsis thaliana and Eutrema salsugineum plants irrigated with water and with $\mathrm{NaCl}$ solution. Salinity of 0.15 and $0.3 \mathrm{M} \mathrm{NaCl}$, was used for $A$. thaliana and $E$. salsugineum, respectively. Data represent means $\pm \mathrm{SD}, n=6(A, B), 3-4(C, D, E) . \diamond-$ a significant difference between control and salinity-treated plants; * - a significant difference from A. thaliana from the same treatment. 


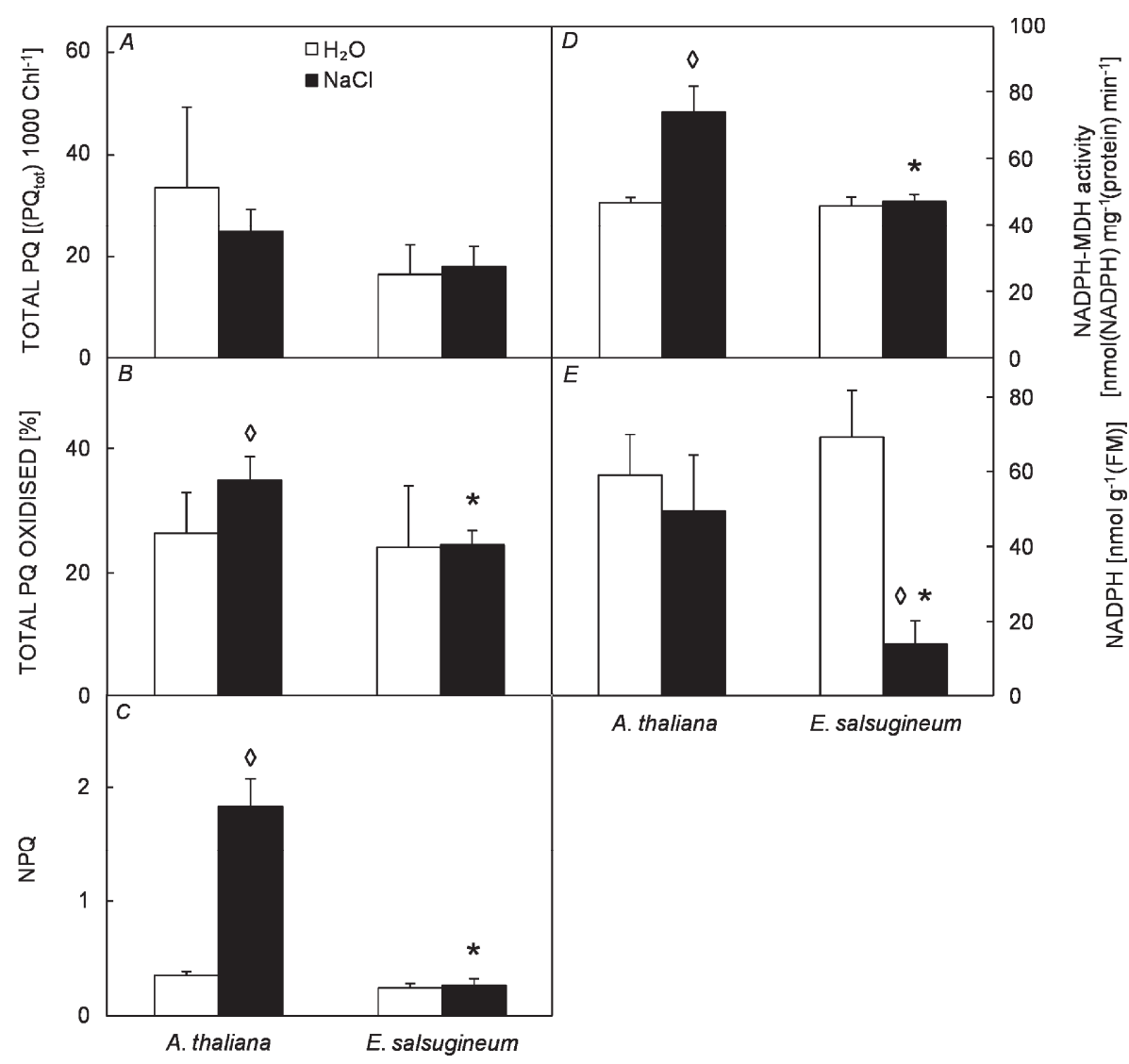

Fig. 2. Effects of salinity on the content of total foliar PQ $(A)$, the redox state of total foliar PQ (oxidized and reduced, $B$ ), NPQ parameter $(C)$, the activity of NADP-MDH $(D)$, and the content of NADPH $(E)$ in leaves of Arabidopsis thaliana and Eutrema salsugineum plants irrigated with water and with $\mathrm{NaCl}$ solution. Salinity of 0.15 and $0.3 \mathrm{M} \mathrm{NaCl}$, was used for $A$. thaliana and E. salsugineum, respectively. Data represent means $\pm \mathrm{SD}, n=3-5(A, B), 6-7(C), 5(D), 3-6(E) . \diamond-$ a significant difference between control and salinity-treated plants; * - a significant difference from $A$. thaliana from the same treatment

Arabidopsis plants, the content of $\alpha$-Toc was twofold compared to that in Eutrema, and this difference increased to threefold under salt conditions. The content of PC-8 was the same in control plants of both species. The content of $\mathrm{PC}-\mathrm{OH}$ was similar in control plants of both species and increased only in $A$. thaliana after salinity.

Salinity-evoked changes in the foliar plastoquinone and NPQ: Change of the redox state of PQ pool is typically observed under stress, however, studies on both of the redox state of the PQ-pool and total PQ (photosynthetically active and inactive) during salinity are rather scarce. Therefore, we analysed the content of total foliar PQ (oxidized and reduced) and its redox state in both species after salinity (Fig. 2A,B). Under control conditions, the total PQ content was similar in both species and maintained mostly in a reduced state $(72.7 \%$ for $A$. thaliana and $75.9 \%$ for $E$. salsugineum). The salinity caused a significant oxidation of total PQ in $A$. thaliana (to $65.4 \%$ ), and at the same time the total PQ amount in relation to $\mathrm{Chl}$ did not change considerably. In turn, in E. salsugineum, salinity did not affect neither the amount of total PQ nor its redox state which remained mostly reduced $(75.5 \%)$.
In the light of a crucial importance of PQ involvement in many physiological processes, we investigated NPQ as a feasible factor that could influence $P Q$ redox state during mild salinity. In order to estimate if NPQ contributes to the PQ-pool oxidation under conditions of mild salinity, we measured this process before and after salinity treatments (Fig. 2C). Under well-watered conditions, NPQ was fairly similar in A. thaliana and E. salsugineum plants. However, the harmful effect of moderate salinity on the photosynthetic capacity of $A$. thaliana was illustrated by the significant increase in NPQ. In contrast, treatment of E. salsugineum with high salinity did not affect the NPQ value that remained as low as in the control plants.

Donor and acceptor site of photosystem I: Salinityinduced stomatal closure reduces an availability of $\mathrm{CO}_{2}$ which is followed by an overaccumulation of NADPH allowing to increase ROS formation, as shown in salttreated leaves of Rumex (Chen et al. 2004). This situation negatively affected PSI due to the limited pool of electron acceptors. In control condition, the efficiency of PSI ( $\left.\mathrm{Y}_{\mathrm{I}}\right)$ was higher in E. salsugineum than that in A. thaliana (Table 1). This was associated by a very low value of 
Table 1. The effect of salinity on the quantum efficiency of PSI ( $\left.\mathrm{Y}_{\mathrm{I}}\right)$, donor-side limitation to PSI ( $\left.\mathrm{Y}_{\mathrm{ND}}\right)$, and acceptor-side limitation to PSI ( $\mathrm{Y}_{\mathrm{NA}}$ ) measured in leaves of Arabidopsis thaliana and Eutrema salsugineum plants irrigated with water and with $\mathrm{NaCl}$ solution. Salinity of 0.15 and $0.3 \mathrm{M} \mathrm{NaCl}$, was used for A. thaliana and E. salsugineum, respectively. Data represent means $\pm \mathrm{SD}(n=7-8)$. "Diamond" indicates a significant difference between control and salinity-treated plants. "Asterisk" indicates a significant difference from A. thaliana from the same treatment.

\begin{tabular}{lllll}
\hline Parameter & $\begin{array}{l}\text { Control } \\
\text { A. thaliana }\end{array}$ & E. salsugineum & $\begin{array}{l}\text { Salinity } \\
\text { A. thaliana }\end{array}$ & E. salsugineum \\
\hline $\mathrm{Y}_{\mathrm{I}}$ & $0.739 \pm 0.081$ & $0.940 \pm 0.040^{*}$ & $0.442 \pm 0.067^{\diamond}$ & $0.941 \pm 0.030^{*}$ \\
$\mathrm{Y}_{\mathrm{ND}}$ & $0.121 \pm 0.061$ & $0.004 \pm 0.002^{*}$ & $0.453 \pm 0.078^{\diamond}$ & $0.003 \pm 0.000^{*}$ \\
$\mathrm{Y}_{\mathrm{NA}}$ & $0.141 \pm 0.004$ & $0.058 \pm 0.035$ & $0.115 \pm 0.019$ & $0.060 \pm 0.033$ \\
\hline
\end{tabular}

donor- and acceptor-side limitations to PSI represented by $\mathrm{Y}_{\mathrm{ND}}$ and $\mathrm{Y}_{\mathrm{NA}}$, respectively. A noteworthy decrease in $\mathrm{Y}_{\mathrm{I}}$ was noted in salinity-treated $A$. thaliana, in concert with a strongly increased $\mathrm{Y}_{\mathrm{ND}}$ and only slightly decreased $\mathrm{Y}_{\mathrm{NA}}$. Whereas, in salinity-treated E. salsugineum, efficiency of PSI remained at high level and a primary electron donor to PSI (P700) was kept at highly reduced state.

To get an insight how the redox state of the PSI acceptors is affected under the conditions of moderate salinity, we compared the enzyme activity of chloroplastic NADP-MDH and the content of NADPH in both species,

\section{Discussion}

There are numerous data on the correlation between an increased antioxidant capacity and salt tolerance (for a recent review see: Ozgur et al. 2013, Bose et al. 2014, Niewiadomska and Wiciarz 2015). Within the antioxidative response, an activation of the ascorbateglutathione cycle plays a major role, as documented for several salt-tolerant glycophytic and halophytic species: Pisum sativum (Hernandez et al. 1995), Lycopershion pennelli (Mittova et al. 2003), Oryza sativa (Vaidyanathan et al. 2003), Suaeda salsa (Cai-Hong et al. 2005), Cakile maritima (Amor et al. 2006), and Thellungiella parvula (Uzilday et al. 2015). Here documented increase in the contents of ascorbate and glutathione in E. salsugineum due to salinity stress is in agreement with this view. As suggested by Bose et al. (2014), in halophytes, an efficient operation of this cycle is crucial for decreasing the amounts of $\mathrm{H}_{2} \mathrm{O}_{2}$ "signatures" required for signalling purposes. In contrast, in $A$. thaliana plants, lack of positive responses to salinity from ascorbate and glutathione may suggest a weak involvement of the ascorbate-glutathione cycle.

However, an opposite salinity stress response was related to the lipophilic antioxidants ( $\alpha$-Toc and PC- 8$)$ and $\mathrm{PC}-\mathrm{OH}$, which increased only in $A$. thaliana. Since PC$\mathrm{OH}$, the hydroxyl derivative of plastochromanol, has been assumed as an indicator of singlet oxygen-mediated oxidative damage (Szymańska et al. 2014), we argued that A. thaliana plants activated an $\alpha$-Toc turnover under a mild salinity in order to counteract the toxicity of ${ }^{1} \mathrm{O}_{2}$. Interestingly, in E. salsugineum, the content of $\alpha$-Toc was rather low, which is in contrast to the data, documenting a before and after salinity treatment (Fig. 2D,E). In control plants, the enzyme activity was similar both in $A$. thaliana and $E$. salsugineum, whereas after salinity the activity of this enzyme was stimulated only in A. thaliana. In turn, a different pattern of responses were observed in the concentration of NADPH under salinity, where $\mathrm{NaCl}$ treatment considerably decreased the content of NADPH in E. salsugineum and did not affect its amount in A. thaliana. These results pointed to the clear change in the maintenance of NADPH under salinity stress in the two species.

constitutively higher amounts of $\alpha$-Toc in salinity-resistant variety of Gossypium hirsutum and halophytic Cakile maritima (Gossett et al. 1994, Ellouzi et al. 2011). This may suggest a very efficient PSII protection in E. salsugineum independent from that provided by $\alpha$-Toc. At least in part, this enhanced protection may be given by cycling of electrons around PSII in concert with an enhanced $\mathrm{H}_{2} \mathrm{O}_{2}$ production, as it was shown before (Stepien and Johnson 2009, Wiciarz et al. 2015).

One of the typical effects of salinity stress is a declined photochemical efficiency of PSII. A high excitation pressure on PSII reduces the PQ-pool and disturbs linear electron transport which results most likely from acidification of the thylakoid lumen which in turn activates the xantophyll cycle (Sacksteder and Kramer 2000). This cycle is involved in a harmless dissipation of excess of absorbed light energy from PSII (Baker 2008). An activation of the xantophyll cycle, visualized by NPQ parameter, in turn lowers the excitation pressure on PSII and minimizes ${ }^{1} \mathrm{O}_{2}$ production within the PSII antenna, and prevents overreduction of the PQ-pool (Müller et al. 2001). However, under high light stress, enhanced singlet oxygen production may influence redox state of the PQ-pool by its fast oxidation (Kruk and Szymańska 2012). Our results support the latter view, since NPQ increased after salinity in A. thaliana and at the same time, a significant oxidation of total PQ was observed. If we also take into account that plastoquinol is an effective singlet oxygen scavenger (Kruk and Trebst 2008) and it is oxidized to PQ and $\mathrm{H}_{2} \mathrm{O}_{2}$ in this reaction (Khorobrykh et al. 2015), it could be 
assumed that production of singlet oxygen in A. thaliana additionally contributes to PQ-pool oxidation.

Halophytic plants seem to use other strategies than NPQ to avoid PSII photoinhibition under salinity. This has been earlier documented for a halophytic $\mathrm{C}_{3}$-CAM intermediate Mesembryanthemum crystallinum (Niewiadomska et al. 2011). Here documented, lack of NPQ increase, as well as of PQ pool oxidation in salinity-treated E. salsugineum additionally confirmed the aforementioned results on a minimal singlet oxygen production at PSII. Our data are in agreement with the results of Degl'Innocenti et al. (2009), where a comparison of glycophytic Hordeum vulgare with a more salinityresistant $H$. maritimum revealed a higher PQ reduction in the latter species both in control plants and after salinity treatment. As documented earlier using EPR method, chloroplasts of E. salsugineum are also capable for a decreased formation of the toxic $\mathrm{O}_{2}{ }^{-}$in relation to less toxic $\mathrm{H}_{2} \mathrm{O}_{2}$ in comparison to $A$. thaliana (Pilarska et al. 2016). We hypothesize that a highly reduced state of PQ in E. salsugineum already under control conditions may create a basis for a high $\mathrm{H}_{2} \mathrm{O}_{2}$ formation, which in turn activates defence mechanisms to prevent the production of a more toxic forms of $\operatorname{ROS}\left(\mathrm{O}_{2}{ }^{-}\right.$and $\left.{ }^{1} \mathrm{O}_{2}\right)$ and to prevent lipid peroxidation. Such a strategy would explain a constitutively enhanced expression of stress genes, repeatedly reported for this species (Taji et al. 2004, Gong et al. 2005, Amtmann 2009).

Stress-driven increase in NPQ is associated with an increase in $\Delta \mathrm{pH}$ across the thylakoid membranes. This, in turn, inhibits cytochrome $b 6 f$ complex leading to the limited electron donation to PSI (Johnson 2011). This was visualized by a highly increased donor-side limitation in salt-treated $A$. thaliana plants. In contrast, E. salsugineum plants maintained an extremely low $\mathrm{Y}_{\mathrm{ND}}$, indicative for a very effective electron donation to PSI (Klughammer and Schreiber 1994) both in control and under salinity, as well as, a highly active PSI. An increased PSI efficiency of this species is in line with our earlier determinations made with oxygen electrode (Wiciarz et al. 2015). Extremely low $\mathrm{Y}_{\mathrm{ND}}$ detected in E. salsugineum seems to be closely linked to a more reduced PQ pool. A decreased $\mathrm{Y}_{\mathrm{ND}}$ due to salinity was documented previously with halophytic M. crystallinum (Niewiadomska et al. 2011). Because our measurements were done with excised leaves, an effect of desiccation on PSII and PSI parameters might also be taken into account. Typically, an increase in NPQ occurs due to stomatal closure. In respect to PSI performance, Peeva et al. (2012) described an acceleration of $\mathrm{P} 700^{+}$dark reduction caused by the activity of NADPH dehydrogenase $(\mathrm{NDH})$ in excised leaves of barley. This is, however, unlikely not to affect PSI in a light-adapted state. Hence, a clear differences in NPQ and in PSI parameters persistent in excised leaves of the two species rather illustrated a specific performance of E. salsugineum. On this basis, we speculate that ability to preserve an efficient electron donation to PSI under stress is one of the key traits for a high salinity resistance. It is tempting to speculate that a stimulation of cycling electron transport at PSI might be responsible for this phenomenon, and verification of this hypothesis is under way.

A clear discrepancy in the functioning of PSI between the two species became less evident as far as the acceptor site of PSI is concerned. However, in E. salsugineum, a tendency to lower $\mathrm{Y}_{\mathrm{NA}}$ was detected. Under salinitydependent stomatal closure and lowered $\mathrm{CO}_{2}$ supply, a restriction occurs in usage of NADPH by Calvin cycle. In this situation, several strategies of processing of an excessive reducing power might be involved. As visualized in our study, at similar levels of FNR protein (Fig. 1S, supplement available online) in A. thaliana and E. salsugineum, different strategies of NADPH management were activated under salinity. In $A$. thaliana, a salinity-dependent increase in the activity of NADPH$\mathrm{MDH}$ may indicate an intensified export of reducing equivalents from chloroplasts via so-called malate valve. This safety valve takes advantage of excess NADPH to convert oxaloacetate to malate which is then transported to cytosol (Scheibe 2004). The activity of this enzyme is regulated by increasing reduction of thioredoxin pool and switches off when NADPH is consumed for assimilatory processes (Scheibe et al. 2005). In contrast, in E. salsugineum, a strong salinity-dependent decline in the content of NADPH may suggest either its limited production or intensified consumption. One explanation of this phenomenon might be an activation of other efficient electron sinks from reduced ferredoxin. A competition for electrons from PSI has been clearly shown with Arabidopsis nadp$m d h$ mutant, in which a set of compensatory mechanisms, such as NTRC/2-Cys Prx system, proline biosynthesis or photorespiration, were activated in order to avoid excess of reducing equivalents (Hebbelmann et al. 2012). In agreement with this view, in E. salsugineum, an activation of alternative sinks for NADPH, such as adenosine-5'phosphosulfate reductase involved in sulfate assimilation (Gong et al. 2005), thioredoxin CDSP32 (M'rah et al. 2007), peroxiredoxins BAS1 and PrxQ (Gao et al. 2009, Pilarska et al. 2016), and proline biosynthesis (Ghars et al. 2012) may be suggested. Another possibility to explain a low NADPH production might be an activation the cyclic electron flow around PSI.

In conclusion, the new features of high salinity resistance of E. salsugineum were resolved in this study: an activation of the ascorbate-glutathione cycle, avoidance of singlet oxygen-mediated damage at PSII, and in the same time, the ability to sustain a highly reduced states of PQ pool and P700. These are, however, associated with a very low NADPH yield. 
Open Access This article is distributed under the terms of the Creative Commons Attribution License which permits any use, distribution, and reproduction in any medium, provided the original author(s) and the source are credited.

\section{References}

Amor N., Jiménez A., Megdiche W. et al.: Response of antioxidant systems to $\mathrm{NaCl}$ stress in the halophyte Cakile maritima. - Physiol. Plantarum 126: 446-457, 2006.

Amtmann A.: Learning from evolution: Thellungiella generates new knowledge on essential and critical components of abiotic stress tolerance in plants. - Mol. Plant. 2: 3-12, 2009.

Apel K., Hirt H.: Reactive oxygen species: metabolism, oxidative stress, and signal transduction. - Annu. Rev. Plant Biol. 55: 373-399, 2004.

Baier M., Dietz K.-J.: Chloroplasts as source and target of cellular redox regulation: a discussion on chloroplast redox signals in the context of plant physiology. - J. Exp. Bot. 56: 1449-1462, 2005.

Baker N.R.: Chlorophyll fluorescence: A probe of photosynthesis in vivo. - Annu. Rev. Plant Biol. 59: 89-113, 2008.

Bilger W., Björkman O.: Role of the xanthophyll cycle in photoprotection elucidated by measurements of light-induced absorbency changes, fluorescence and photosynthesis in leaves of Hedera canariensis. - Photosynth. Res. 25: 173-185 1990.

Bose J., Rodrigo-Moreno A., Shabala S.: ROS homeostasis in halophytes in the context of salinity stress tolerance. - J. Exp. Bot. 65: 1241-1257, 2014.

Chang C.C., Ball L., Fryer M.J. et al.: Induction of ascorbate peroxidase 2 expression in wounded Arabidopsis leaves does not involve known wound-signalling pathways but is associated with changes in photosynthesis. - Plant J. 38: 499511,2004

Chen H.-X., Gao H.-Y., An S.-Z., Li W.-J.: Dissipation of excess energy in Mehler-peroxidase reaction in Rumex leaves during salt shock. - Photosynthetica 42: 117-122, 2004.

Dassanayake M., Oh D., Hong H. et al.: Transcription strength and halophytic lifestyle. - Trend. Plant Sci. 16: 1-3, 2011.

Degl'Innocenti E., Hafsi C., Guidi L. et al.: The effect of salinity on photosynthetic activity in potassium-deficient barley species. - J. Plant Physiol. 166: 1968-1981, 2009.

Dietzel L., Bräutigam K., Pfannschmidt T.: Photosynthetic acclimation: state transitions and adjustment of photosystem stoichiometry - functional relationships between short-term and long-term light quality acclimation in plants. - FEBS J. 275: 1080-1088, 2008.

Ellouzi H., Hamed K.B., Cela J. et al.: Early effects of salt stress on the physiological and oxidative status of Cakile maritima (halophyte) and Arabidopsis thaliana (glycophyte). - Physiol. Plantarum 142: 128-143, 2011.

Foyer C.H., Noctor G.: Ascorbate and glutathione: the heart of the redox hub. - Plant Physiol. 155: 2-18, 2011.

Foyer C.H., Noctor G.: Redox regulation in photosynthetic organisms: signaling, acclimation, and practical implications. Antioxid. Redox Sign. 11: 861-905, 2009.

Gao F., Zhou Y., Zhu W.: Proteomic analysis of cold stressresponsive proteins in Thellungiella rosette leaves. - Planta 230: 1033-1046, 2009.

Ghars M.A., Richard L., Lefebvre-De Vos D.: Phospholipases C and $\mathrm{D}$ modulate proline accumulation in Thellungiella halophila/salsuginea differently according to the severity of salt or hyperosmotic stress. - Plant Cell Physiol. 53: 183-192, 2012.
Gong Q., Li P., Ma S.: Salinity stress adaptation competence in the extremophile Thellungiella halophila in comparison with its relative Arabidopsis thaliana: Salinity stress adaptation in T. halophila. - Plant J. 44: 826-839, 2005.

Gossett D., Millhollon E., Lucas M.: Antioxidant response to $\mathrm{NaCl}$ stress in salt-tolerant and salt-sensitive cultivars of cotton. - Plant Cell Rep. 13: 706-714, 1994.

Gruszka J., Pawlak A., Kruk J.: Tocochromanols, plastoquinol and other biological prenyllipids as singlet oxygen quenchers determination of singlet oxygen quenching rate constants and oxidation products. - Free Radical Biol. Med. 45: 920-928, 2008.

Hebbelmann I., Selinski J., Wehmeyer C. et al.: Multiple strategies to prevent oxidative stress in Arabidopsis plants lacking the malate valve enzyme NADP-malate dehydrogenase. - J. Exp. Bot. 63: 1445-1459, 2012.

Hernández J., Olmos E., Corpas F. et al.: Salt-induced oxidative stress in chloroplasts of pea plants. - Plant Sci. 105: 151-167, 1995.

Johnson G.N.: Physiology of PSI cyclic electron transport in higher plants. - Biochim. Biophys. Acta. 1807: 384-389, 2011.

Khorobrykh S.A., Karonen M., Tyystjärvi E.: Experimental evidence suggesting that $\mathrm{H}_{2} \mathrm{O}_{2}$ is produced within the thylakoid membrane in a reaction between plastoquinol and singlet oxygen. - FEBS Lett. 589: 779-786, 2015.

Klughammer C., Schreiber U.: An improved method, using saturating light pulses, for the determination of photosystem I quantum yield via $\mathrm{P} 700^{+}$-absorbance changes at $830 \mathrm{~nm}$. Planta 192: 261-268, 1994.

Kruk J., Karpinski S.: An HPLC-based method of estimation of the total redox state of plastoquinone in chloroplasts, the size of the photochemically active plastoquinone-pool and its redox state in thylakoids of Arabidopsis. - Biochim. Biophys. Acta 1757: 1669-1675, 2006

Kruk J., Trebst A.: Plastoquinol as a singlet oxygen scavenger in photosystem II. - Biochim. Biophys. Acta 1777: 154-162, 2008.

Kruk J., Szymańska R.: Singlet oxygen and non-photochemical quenching contribute to oxidation of the plastoquinone-pool under high light stress in Arabidopsis. - BBA-Bioenergetics 1817: 705-710, 2012.

Kruk J., Szymańska R., Nowicka B. et al.: Function of isoprenoid quinones and chromanols during oxidative stress in plants. New Biotechnol. 33: 636-643, 2016.

Lichtenthaler H.K.: Chlorophylls and carotenoids: pigments of photosynthetic biomembranes. - Methods Enzymol. 148: 350382, 1987.

Lokhande V.H., Srivastava A.K., Srivastava S. et al.: Regulated alterations in redox and energetic status are the key mediators of salinity tolerance in the halophyte Sesuvium portulacastrum (L.) L. - Plant Growth Regul. 65: 287-298, 2011.

Luwe M.W.F., Takahama U., Heber U.: Role of ascorbate in detoxifying ozone in the apoplast of spinach (Spinacia oleracea L.) leaves. - Plant Physiol. 101: 969-976, 1993.

Mittova V., Theodoulou F.L., Kiddle G. et al.: Coordinate induction of glutathione biosynthesis and glutathione- 
metabolizing enzymes is correlated with salt tolerance in tomato. - FEBS Lett. 554: 417-421, 2003.

M'rah S., Ouerghi Z., Eymery F.: Efficiency of biochemical protection against toxic effects of accumulated salt differentiates Thellungiella halophila from Arabidopsis thaliana. - J. Plant Physiol. 164: 375-384, 2007.

Müller P., Li X.P., Niyogi K.K.: Non-photochemical quenching. A response to excess light energy. - Plant Physiol. 125: 1558$1566,2001$.

Munné-Bosch S.: The role of alpha-tocopherol in plant stress tolerance. - J. Plant Physio. 162: 743-748, 2005.

Munné-Bosch S., Alegre L.: The function of tocopherols and tocotrienols in plants. - Crit. Rev. Plant Sci. 21: 31-57, 2002.

Munns R., Tester M.: Mechanisms of salinity tolerance. - Annu. Rev. Plant Biol. 59: 651-681, 2008.

Murata N., Takahashi S., Nishiyama Y. et al.: Photoinhibition of photosystem II under environmental stress. - BBABioenergetics 1767: 414-421, 2007.

Niewiadomska E., Bilger W., Gruca M. et al.: CAM-related changes in chloroplastic metabolism of Mesembryanthemum crystallinum L. - Planta 233: 275-285, 2011.

Niewiadomska E., Wiciarz M.: Adaptations of chloroplastic metabolism in halophytic plants. - In: Lüttge U., Beyschlag W. (ed.): Progress in Botany. Pp. 177-193. Springer International Publishing Switzerland 2015.

Noctor G.: Metabolic signalling in defence and stress: the central roles of soluble redox couples. - Plant Cell Environ. 29: 409425, 2006

Noctor G., Foyer C.H.: Ascorbate and glutathione: keeping active oxygen under control. - Annu. Rev. Plant Phys. 49: 249-279, 1998.

Nowicka B., Kruk J.: Occurrence, biosynthesis and function of isoprenoid quinones. - BBA-Bioenergetics 1797: 1587-1605, 2010.

Ozgur R., Uzilday B., Sekmen A.H. et al.: Reactive oxygen species regulation and antioxidant defence in halophytes. Funct. Plant Biol. 40: 832-847, 2013.

Pang C.H., Zhang S.J., Gong Z.Z. et al.: $\mathrm{NaCl}$ treatment markedly enhances $\mathrm{H}_{2} \mathrm{O}_{2}$-scavenging system in leaves of halophyte Suaeda salsa. - Physiol. Plantarum 125: 490-499, 2005.

Peeva V.N., Tóth S.Z., Cornic G., Ducruet J.-M.: Thermoluminescence and P700 redox kinetics as complementary tools to investigate the cyclic/chlororespiratory electron pathways in stress conditions in barley leaves - Physiol. Plantarum 144: 8397, 2012.

Pfalz J., Liebers M., Hirth M. et al.: Environmental control of plant nuclear gene expression by chloroplast redox signals. Front. Plant Sci. 3: 257, 2012.

Pfannschmidt T.: Chloroplast redox signals: how photosynthesis controls its own genes. - Trends Plant Sci. 8: 33-41, 2003.

Pfannschmidt T., Bräutigam K., Wagner R. et al.: Potential regulation of gene expression in photosynthetic cells by redox and energy state: approaches towards better understanding. Ann. Bot.-London 103: 599-607, 2009.

Pilarska M., Wiciarz M., Jajić I. et al.: A different pattern of production and scavenging of reactive oxygen species in halopythic Eutrema salsugineum (Thellungiella salsuginea) plants in comparison to Arabidopsis thaliana and its relation to salt stress signaling. - Front. Plant. Sci. 7: 1179, 2016.

Pospíšil P.: Production of reactive oxygen species by photosystem II. - BBA-Bioenergetics 1787: 1151-1160, 2009.

Puthiyaveetil S., Ibrahim I.M., Allen J.F.: Oxidation-reduction signalling components in regulatory pathways of state transitions and photosystem stoichiometry adjustment in chloroplasts. - Plant Cell Environ. 35: 347-359, 2012.

Sacksteder C.A., Kramer D.M.: Dark-interval relaxation kinetics (DIRK) of absorbance changes as a quantitative probe of steadystate electron transfer. - Photosynth. Res. 66: 145-158, 2000.

Scheibe R., Stitt M.: Comparison of NADP-malate dehydrogenase activation, $\mathrm{Q}_{A}$ reduction and $\mathrm{O}_{2}$ evolution in spinach leaves. - Plant Physiol. Bioch. 26: 473-481, 1988.

Scheibe R.: Malate valves to balance cellular energy supply. Physiol. Plantarum 120: 21-26, 2004.

Scheibe R., Backhausen J.E., Emmerlich V. et al.: Strategies to maintain redox homeostasis during photosynthesis under changing conditions. - J. Exp. Bot. 56: 1481-1489, 2005.

Ślesak I., Karpinska B., Surówka E. et al.: Redox changes in the chloroplast and hydrogen peroxide are essential for regulation of C(3)-CAM transition and photooxidative stress responses in the facultative CAM plant Mesembryanthemum crystallinum $\mathrm{L}$. - Plant Cell Physiol. 44: 573-581, 2003.

Stepien P., Johnson G.N.: Contrasting responses of photosynthesis to salt stress in the glycophyte Arabidopsis and the halophyte Thellungiella: role of the plastid terminal oxidase as an alternative electron sink. - Plant Physiol. 149: 1154-1165, 2009.

Suzuki N., Koussevitzky S., Mittler R., Miller G.: ROS and redox signalling in the response of plants to abiotic stress. - Plant Cell Environ. 35: 259-270, 2012.

Szymańska R., Kruk J.: Plastoquinol is the main prenyllipid synthesized during acclimation to high light conditions in Arabidopsis and is converted to plastochromanol by tocopherol cyclase. - Plant Cell Physiol. 51: 537-545, 2010.

Szymańska R., Nowicka B., Kruk J.: Hydroxy-plastochromanol and plastoquinone-C as singlet oxygen products during photooxidative stress in Arabidopsis. - Plant Cell Environ. 37: 14641473,2014

Taji T., Seki M., Satou M. et al.: Comparative genomics in salt tolerance between Arabidopsis and Arabidopsis-related halophyte salt cress using Arabidopsis microarray. - Plant Physiol. 135: 1697-1709, 2004

Takahama U., Oniki T.: Regulation of peroxidase-dependent oxidation of phenolics in the apoplast of spinach leaves by ascorbate. - Plant Cell Physiol. 33: 379-387, 1992.

Takahashi S., Murata N.: How do environmental stresses accelerate photoinhibition? - Trends Plant Sci. 13: 178-182, 2008.

Takahashi S., Murata N.: Glycerate-3-phosphate, produced by $\mathrm{CO}_{2}$ fixation in the Calvin cycle, is critical for the synthesis of the D1 protein of photosystem II. - Biochim. Biophys. Acta 1757: 198-205, 2006.

Uzilday B., Ozgur R., Sekmen A.H.: Changes in the alternative electron sinks and antioxidant defence in chloroplasts of the extreme halophyte Eutrema parvulum (Thellungiella parvula) under salinity. - Ann. Bot.-London 115: 449-463, 2015.

Vaidyanathan H., Sivakumar P., Chakrabarty R. et al. Scavenging of reactive oxygen species in $\mathrm{NaCl}$-stressed rice (Oryza sativa L.). - differential response in salt-tolerant and sensitive varieties. - Plant Sci. 165: 1411-1418, 2003.

Vinocur B., Altman A.: Recent advances in engineering plant tolerance to abiotic stress: achievements and limitations. Curr. Opin. Biotech. 16: 123-132, 2005.

Wiciarz M., Gubernator B., Kruk J. et al.: Enhanced chloroplastic generation of $\mathrm{H}_{2} \mathrm{O}_{2}$ in stress-resistant Thellungiella salsuginea in comparison to Arabidopsis thaliana. - Physiol. Plantarum 153: 467-476, 2015. 